Le capital au XXlème siècle

\section{T. Piketty}

\section{París: Seuil, 976.}

El economista Thomas Piketty ha publicado su último libro, titulado $L e$ capital au xxième siècle, en la colección «Les livres du nouveau monde», dirigida por Pierre Rosanvallon. Piketty es uno de los economistas europeos más precoces y brillantes de su generación, tanto su obra como su trayectoria académica dan cuenta de ello. Tras realizar sus estudios superiores en la prestigiosa Escuela Normal Superior (ENS), con tan solo 22 años defendió su tesis de economía en la ENS y la London School of Economics, trabajo por el que obtuvo el premio de la mejor tesis, concedido por la Asociación Francesa de Ciencias Económicas. Entre 1993 y 1995, fue profesor en el Massachussets Institute of Technology (MIT) de Boston, antes de integrar el CNRS francés. En 2000, fue nombrado director de estudios de la Escuela de Altos Estudios en Ciencias Sociales (EHESS) y, entre 2005 y 2007, fue el primer director de la Escuela de Economía de París, de la que es también catedrático.

A lo largo de estos años, ha dedicado la mayor parte de sus investigaciones y reflexiones a la dinámica histórica de la renta y del patrimonio, buena parte de estas investigaciones han sido llevadas a cabo en colaboración, especialmente, con Anthony Atkinson y Emmanuel Saez. Tras publicar Les hauts revenus en France au XXème siècle en 2001, extendió su trabajo al ámbito internacional. Gracias a su colaboración con Atkinson, ha publicado dos libros, en 2007 (Atkinson y Pyketty, 2007) y 2010 (Atkinson y Pyketty, 2010), que cubren 20 países, constituyendo así la más amplia base de datos disponible sobre la evolución histórica de las desigualdades de renta. Asimismo, se ha interesado por la teoría de la imposición óptima de la renta y del capital. Le capital au Xxième siècle está 
igualmente profundamente influido por Gilles Postel-Vinay y Jean-Laurent Rosenthal y por las investigaciones históricas que siguen llevando a cabo sobre los archivos sucesorios parisinos desde la época de la Revolución francesa hasta la actualidad.

En su obra, el joven economista galo delimita geográfica e históricamente su objeto de estudio: intenta analizar la dinámica del reparto de la riqueza a nivel mundial, tanto dentro de cada país como entre países, desde el siglo xvin hasta la actualidad. No obstante, en la práctica, las múltiples limitaciones sobre los datos disponibles le han obligado en ciertas ocasiones a reducir el ámbito de estudio. Asimismo, presta una atención particular al caso francés, básicamente por la antigüedad, variedad y accesibilidad de las fuentes. De hecho, la Revolución francesa puso en marcha un incomparable observatorio de las fortunas: el sistema de registro del patrimonio terrenal e inmobiliario instaurado en los años 1790-1800 es especialmente moderno y universal para la época y explica por qué las fuentes sucesorias francesas son especialmente ricas.

Piketty constata que el reparto de la riqueza constituye una de las cuestiones más debatidas actualmente, aunque nuestro conocimiento sobre su evolución a largo plazo sea limitado. Piketty se pregunta en su libro si la dinámica de la acumulación de capital privado conduce inevitablemente a la concentración cada vez superior de la riqueza y del poder en las manos de algunos, o si las fuerzas reequilibrantes del crecimiento, de la competencia y del avance técnico conducen espontáneamente a la reducción de las desigualdades y a una estabilización en las fases más avanzadas del desarrollo. En definitiva, a lo largo de las 976 páginas que componen su libro, Piketty intenta contestar a la siguiente pregunta: ¿qué sabemos realmente sobre la evolución de la distribución de la renta y del patrimonio desde el siglo xviri, y qué lecciones podemos extraer de ello para el siglo $\mathrm{xxI}$ ?

Aun reconociendo que sus respuestas son imperfectas e incompletas, subraya que su estudio se basa en datos históricos y comparativos mucho más extensos que todos los trabajos realizados anteriormente y emplea un marco teórico renovado que permite mejorar nuestra comprensión de las tendencias y mecanismos vigentes. Defiende la hipótesis según la cual, a partir del momento en el que la tasa de beneficio del capital supera duraderamente la tasa de crecimiento de la producción y de la renta, como ocurría en el siglo XVIII y corre el riesgo de ocurrir de nuevo en el siglo xxi, el capitalismo produce mecánicamente unas desigualdades que cuestionan los valores meritocráticos sobre los que se fundamentan las sociedades democráticas (Piketty, 2013: 16). 
Para demostrar su tesis, el economista galo recurre a dos tipos de fuentes: las que aluden a la renta y a la desigualdad de su distribución, y las que se refieren al patrimonio, a su reparto y al vínculo que mantienen con la renta. En el primer caso, su labor consiste en extender a una escala espacial y temporal superior el trabajo novedoso y pionero de Kuznets (1953), que medía la evolución de las desigualdades de renta en Estados Unidos de 1913 a 1948. Esta extensión permite poner en perspectiva las evoluciones constatadas por Kuznets (1955) y conduce a cuestionar el vínculo optimista que establece entre desarrollo económico y reparto de la riqueza. Intenta utilizar las mismas fuentes, los mismos métodos y los mismos conceptos para todos los países estudiados. En el segundo caso, reúne todos los datos disponibles sobre el patrimonio, su reparto $y$ la relación que mantienen con la renta, teniendo en cuenta que el patrimonio juega un papel importante sobre la renta a través de la renta del patrimonio. En ese sentido, conviene recordar que la renta consta de dos aspectos: las rentas del trabajo y las rentas del capital. Los datos de la WTID ofrecen mucha información sobre la evolución de las rentas del capital a lo largo del siglo $\mathrm{xx}$.

Sobre la base de estos datos, Piketty extrae dos conclusiones fundamentales. Por una parte, considera que es preciso desconfiar de cualquier de- terminante económico en esta materia, ya que la historia del reparto de la riqueza es siempre una historia profundamente política y no puede reducirse a mecanismos meramente económicos. Especialmente la reducción de las desigualdades observadas en los países desarrollados entre los años 1900-1910 y 1950-1960 es ante todo el producto de las guerras y de las políticas públicas implementadas tras estos dramas. Asimismo, el incremento de las desigualdades desde los años 1970-1980 resulta en gran medida de los cambios políticos acontecidos a lo largo de las últimas décadas, sobre todo en materia fiscal y financiera (Piketty, 2013: 47). Por otra parte, el economista galo estima que la dinámica del reparto de la riqueza pone en juego poderosos mecanismos que empujan alternativamente hacia la convergencia o hacia la divergencia, y que no existe ningún proceso natural y espontáneo que permita evitar que las tendencias desestabilizadoras y fuente de desigualdades se impongan duraderamente.

Sin embargo, Piketty amplía su reflexión a la ciencia económica, dado que considera que la disciplina económica es prisionera de su pasión por las matemáticas y las especulaciones teóricas e incluso ideológicas, en detrimento de la investigación histórica y de la aproximación a las demás ciencias sociales (Piketty, 2013:63). Considera que a menudo los economistas están preocupados ante todo por problemas 
matemáticos que solo les interesan a ellos mismos, lo que les permite dar una apariencia de cientificidad y evitar contestar a preguntas mucho más complejas provenientes de la sociedad que los rodea. A ese respecto, observa una diferencia entre Estados Unidos y Europa, donde los economistas no gozan del mismo prestigio y se ven obligados a abrirse a otras disciplinas y a la sociedad. En ese sentido, observa que los economistas europeos no muestran la arrogancia y la certeza de ser superiores a los demás que muestran ciertos economistas norteamericanos; Piketty reconoce que cuando se encontraba en el MIT, su sueño era ser director de estudios en la EHESS, adonde fueron profesores, sociólogos, historiadores y antropólogos como Lucien Febvre, Fernand Braudel, Claude Lévi-Strauss o Pierre Bourdieu.

Piketty considera que los economistas no deben esforzarse por alejarse de las demás ciencias sociales y que la ciencia económica solo podrá desarrollarse gracias a un diálogo fructífero con ellas. Observa que numerosos economistas desconocen buena parte de la producción científica en sociología, historia o antropología, lo que les ha llevado a no prestar la atención suficiente a la dinámica histórica del reparto de la riqueza y la estructura de clases (Piketty, 2013: 65). Estima que conviene ser pragmático y movilizar métodos y enfoques provenientes de otras disciplinas, en lugar de limitarse a las perspectivas estrictamente económicas; "hay que partir de las cuestiones de fondo e intentar responder a estas preguntas» (Piketty, 2013: 65). Subraya que su trabajo ha consistido inicialmente en reunir fuentes $y$ en establecer hechos históricos sobre el reparto de la renta y del patrimonio, antes de recurrir a teorías, modelos y conceptos abstractos, pero intenta hacerlo con parsimonia, es decir, únicamente en la medida en que la teoría permite una mejor comprensión de las evoluciones estudiadas.

El libro de Piketty se divide en cuatro partes y se subdivide en 16 capítulos. La primera parte, titulada «Renta y capital», introduce las nociones fundamentales que serán abundantemente utilizadas a lo largo del libro. Especialmente, el primer capítulo presenta los conceptos de renta nacional, capital y relación capital/renta y seguidamente describe las grandes líneas de evolución de la distribución de la renta y de la producción. El segundo capítulo analiza más detalladamente la evolución de la tasa de crecimiento de la población y de la producción desde la revolución industrial (Piketty, 2013: 66).

La segunda parte, titulada «La dinámica de la relación capital/renta», tiene como objetivo analizar la manera como se presenta en el inicio del siglo xxi la cuestión de la evolución a largo plazo de la relación capital/renta y del reparto global de la renta del trabajo y de la renta del capital. El tercer 
capítulo presenta las metamorfosis del capital desde el siglo xvin, empezando por los casos de Reino Unido y Francia, mientras que el quinto capítulo introduce los casos de Alemania y Estados Unidos (Piketty, 2013: 66). Los capítulos quinto y sexto extienden geográficamente estos análisis al conjunto del planeta, en la medida en que lo permiten las fuentes y de cara a extraer las conclusiones pertinentes de estas experiencias históricas para analizar la evolución posible de la relación capital/renta y del reparto capital/trabajo en las décadas futuras.

La tercera parte, titulada «La estructura de las desigualdades», empieza familiarizando al lector sobre la desigualdad del reparto de las rentas del trabajo, por una parte, y la propiedad del capital y de la renta que provienen de él, por otra parte. Posteriormente, el octavo capítulo analiza la dinámica histórica de estas desigualdades, empezando por la comparación de los casos francés y norteamericano. Los capítulos noveno y décimo extienden estos análisis al conjunto de los países para los que se dispone de datos históricos, examinando separadamente las desigualdades ante el trabajo y el capital (Piketty, 2013: 67). El capítulo siguiente estudia la evolución de la importancia de la herencia a largo plazo y el capítulo duodécimo analiza las perspectivas de evolución del reparto mundial del patrimonio a lo largo de las primeras décadas del siglo XXI.
La cuarta y última parte, titulada «Regular el capital en el siglo xxI», intenta extraer las lecciones políticas y normativas de las partes anteriores, cuyo objetivo es ante todo establecer los hechos y comprender las razones de las evoluciones observadas. El capítulo decimotercero intenta dibujar los contornos de lo que podría ser un Estado social adecuado al nuevo siglo. El capítulo siguiente propone repensar el impuesto progresivo sobre la renta a la luz de las experiencias pasadas y de las tendencias recientes (Piketty, 2013: 67). El capítulo decimoquinto describe lo que podría ser un impuesto progresivo sobre el capital adecuado al capitalismo patrimonial del siglo XXI, y compara este instrumento ideal con otras modalidades de regulación susceptibles de aparecer, desde el impuesto europeo sobre la fortuna hasta el control del capital al estilo chino, pasando por la inmigración al estilo norteamericano o al retorno generalizado al proteccionismo. El último capítulo trata la cuestión de la deuda pública y de la acumulación óptima del capital público, en un contexto de posible deterioro del capital natural.

En definitiva, Le capital au xxième sièlce es una obra documentada, sólida, ambiciosa e innovadora: documentada, por la variedad y amplitud de los datos movilizados; sólida, por el rigor científico de la demostración, de la utilización de los datos y de su comparación; ambiciosa, por la amplitud temporal 
(desde el siglo xviri hasta la actualidad) y geográfica (la totalidad del planeta) del estudio, e innovadora, tanto por la problemática planteada como por las hipótesis y, posteriormente, las conclusiones formuladas. Está llamada a ser una de las obras de referencia de la economía política en general y de la evolución del reparto de la renta y del patrimonio en particular. Cumple las expectativas generadas por las obras anteriores y por la trayectoria académica de Piketty, que será, sin lugar a dudas, uno de los mejores economistas de los próximos años a nivel mundial.

Para matizar esta valoración positiva, conviene realizar ciertas observaciones. Por una parte, se echa en falta, aunque no sea el objeto del libro, una exposición más extensa y sistematizada de su visión de la ciencia económica y de la orientación que debería predominar tanto en la investigación como en la docencia en esta disciplina. Sería interesante que pudiera dedicar una obra específica a su visión de la economía, que resultaría muy útil para ciertos economistas encerrados en la matematización, la abstracción y la modelización que los alejan de la realidad socioeconómica y los hacen incapaces de tener una representación global, compleja y realista de los fenómenos estudiados. Por otra parte, a pesar del talante pedagógico del autor, el libro ganaría si se redujera su extensión y se recurriera a un lenguaje menos técnico. Por último, esta obra carece de una bibliografía que recoja los libros y artículos citados a lo largo de las 976 páginas que la componen.

Desde su publicación, este libro ha conocido un destino inusual, ya que se ha convertido en un éxito de librerías con la venta de 150.000 ejemplares en Francia y de 450.000 ejemplares en Estados Unidos. Asimismo, ha recibido los elogios de tres premios Nobel de Economía norteamericanos, tales como Joseph Stiglitz, Robert Solow y Paul Krugman; en un artículo publicado en la New York Review of Books, este último ha escrito que Le capital au xxième siècle es «un libro que va a cambiar a la vez la manera en que pensamos las sociedades y la de hacer economía», y añade que se trata de uno de los mejores libros de economía de los últimos diez años. No en vano, ciertos economistas galos, entre los que encontramos a Michel Husson, Robert Boyer o Xavier Timbeau, subrayan la insuficiente teorización de los hechos analizados por Piketty.

\section{Bibliografía}

Atrinson, A. y Piketty, T. (2007): Top Incomes Over the Twentieth Century: A Contrast Between Continental European and Englishspeaking Countries. Oxford: Oxford University Press.

Atrinson, A. y Piketty, T. (2010): Top Incomes: A Global Perspective. Oxford: Oxford University Press. 
Kuznets, S. (1953): Shares of Upper

Income Groups in Income and Savings. New York: NBER.

Kuznets, S. (1955): «Economic Growth and income inequality», The American Economic Review, vol. XLV, n. ${ }^{\circ} 1$, pp. 1-28.

Piketty, T. (2001): Les Hauts Revenus en France au XXème siècle. Inégalités et redistributions, 19011988. Paris: Grasset.

Piketry, T. (2013): Le capital au XXIème siècle. Paris: Seuil.

Eguzki URTEAGA

Diplomacia Pública y Place Branding: el estado de la Marca España Francisco Javier Hernández Alonso 2012. La Coruña. Editorial Netbiblo.

El objetivo que persigue el libro Diplomacia Pública y Place Branding: el estado de la Marca España es el análisis de la diplomacia pública y el place branding como nuevas formas de poder que pueden ejercer los Estados. Se trata de un poder estratégico, conocido como poder blando, que se desarrolla en un escenario internacional y que constituye un poder capaz de influenciar y crear situaciones que sean beneficiosas para los Estados, y mejorar su posicionamiento y competitividad internacional.
En este libro, el doctor Francisco Javier Hernández Alonso, profesor de Pensamiento Político Contemporáneo y Sociología, analiza en profundidad los fenómenos del place branding y las tareas de la diplomacia pública, desmenuzando ambos conceptos y aplicando una visión integradora y complementaria de ambos, como herramientas necesarias en la construcción de la identidad e imagen de los países en la esfera internacional.

El libro se estructura en nueve capítulos, que se dividen en tres secciones fundamentalmente. La primera sección presenta un planteamiento introductorio a los fenómenos objeto de estudio en esta obra, delimita el marco teórico aplicable, especifica los principales actores y define la identidad como principal valor relacional entre ellos. La segunda sección se centra en el concepto de diplomacia pública y su valor estratégico. La tercera y última sección está dedicada a la gestión de la marca, analizando el caso concreto de la Marca España, y concluye que es necesaria la coexistencia y complementariedad de ambos modelos, la diplomacia pública y el place branding.

Un análisis más pormenorizado de los contenidos de esta obra nos lleva a colocar la comunicación como piedra angular para posicionar los Estados en el escenario internacional. La comunicación, en este sentido, se puede entender como «una herramienta para la política, que permite generar inte- 